\title{
Demonstration of Radio-over-Fiber-supported 60 GHz MIMO using Separate Antenna-Pair Processing
}

\author{
Usman Habib ${ }^{1}$, Anthony E Aighobahi ${ }^{1}$, Terence Quinlan ${ }^{2}$, Stuart D Walker ${ }^{2}$ and Nathan J Gomes ${ }^{1}$ \\ ${ }^{1}$ Communications Research Group, University of Kent, Canterbury, UK \\ ${ }^{2}$ School of Computer Science and Electronic Engineering, University of Essex, UK \\ uh23@kent.ac.uk
}

\begin{abstract}
Coverage at millimeter-wave $(\mathrm{mmW})$ frequencies is a constraining bottleneck. Spatial diversity and spatial multiplexing multiple-input multiple-output (MIMO) improve performance over a spread of user locations and these can benefit from wider antenna spacing. Radio-over-Fiber (RoF) transport provides flexibility in deploying a number of widely-spaced Remote Antenna Units (RAUs) connected to the same Central Unit (CU). Hence, mmW systems with an integrated analog RoF fronthaul are strong candidates for use in future $5 \mathrm{G}$ networks. An approach to measure channel coefficients individually for MIMO processing has been demonstrated in a RoF transported $2 \times 2$ MIMO system at $60 \mathrm{GHz}$. Experimental results verify this approach through real $2 \times 2$ experiments.
\end{abstract}

Keywords-Multiple Input Multiple Output MIMO, line of Sight, SISO, EVM

\section{INTRODUCTION}

Radio over Fiber (RoF) fronthaul provides a simple, flexible and low-cost means for interconnecting Remote Antenna Units (RAUs), which are expected to be larger in number in future millimeter wave $(\mathrm{mmW})$ communication systems. RoF-based access can provide centralized control for multiple RAUs. Analog RoF transmission is more suitable for supporting high data rate transmission over $\mathrm{mmW}$ than its digital counterpart because it offers low latency, low loss and does not include costly D/A convertors and synchronization mechanisms [1].

Within the mmW spectrum, the $60 \mathrm{GHz}$ unlicensed band has attracted much interest in recent research, e.g., for WiGig [2]. The Line-of-Sight (LOS) based operation of $60 \mathrm{GHz}$ puts a limit on the coverage capabilities of such systems and performance can degrade significantly as the mobile user changes location.

Multiple-Input Multiple-Output (MIMO) has been adopted in many current standards such as IEEE 802.11ac [3] to satisfy the increase in demand for capacity and reliability in wireless communication systems. Coverage issues where channel propagation effects limit the performance of Single-Input Single-Output (SISO) transmission are common in $\mathrm{mmW}$ communication, which suffers from high free-space transmission loss. MIMO techniques can be useful in these cases to utilize spatial diversity or to multiplex multiple data streams, resulting in an improvement in the performance of the system. This makes MIMO processing a promising candidate to address the issue of coverage for mmW systems. Recent studies have shown that mmW MIMO operation based on LOS communication can provide decorrelation among channels, with sufficient amount of spacing between the transmit antennas [4], as compared to conventional MIMO which heavily relies on rich scattering environment. Spatial diversity and spatial multiplexing techniques can then be applied to achieve MIMO gain, resulting in an improvement in the performance.

Channel measurements in a MIMO based system, and the subsequent signal processing, can be performed by taking measurements for individual channel paths [5,6]. Measurements can be taken by activating only one antenna pair at a time in a proposed MIMO transmission system [7,8]. In [9] we demonstrated improvement in the performance of RoFtransported $60 \mathrm{GHz}$ communications at various user locations, through emulation of MIMO processing in this way, and then examined the performance gain that can be achieved through MIMO assuming the channels were independent. In this work, verification has been done in order to prove the validity of the emulated approach for the RoF based $60 \mathrm{GHz}$ setup. It has been shown here that after both RoF transport and $60 \mathrm{GHz}$ transmission, the channel measurements were stable. This implies that such a measurement approach could be extended to predicting the performance of mmW MIMO processing with larger numbers of antennas/RAUs where there might be hardware limitations during research and development. Results have been verified by conducting the measurements with the setup having two $60 \mathrm{GHz}$ transmit data streams. EVM analysis of the MIMO processing versus SISO is presented to show the improvement in the performance by MIMO processing through multiplexing or spatial diversity.

\section{MIMO EMULATION USING INDIVIDUAL ANTENNA PAIRS}

The experimental setup considered for the first part of this work is shown within the dashed lines in Fig. 1 where an OFDM signal (512 point IFFT, $1 / 8$ cyclic prefix) with $305 \mathrm{MHz}$ bandwidth is generated and is converted to an analog waveform at an Intermediate Frequency IF of $1.5 \mathrm{GHz}$ using an Arbitrary Waveform Generator AWG (Tektronix 7122C). The OFDM 16QAM signal is modulated onto a DFB laser, and transported through a $2.2 \mathrm{~km}$ single-mode fiber (SMF) link to the Remote Antenna Unit (RAU). After detection at the photodiode, the IF 


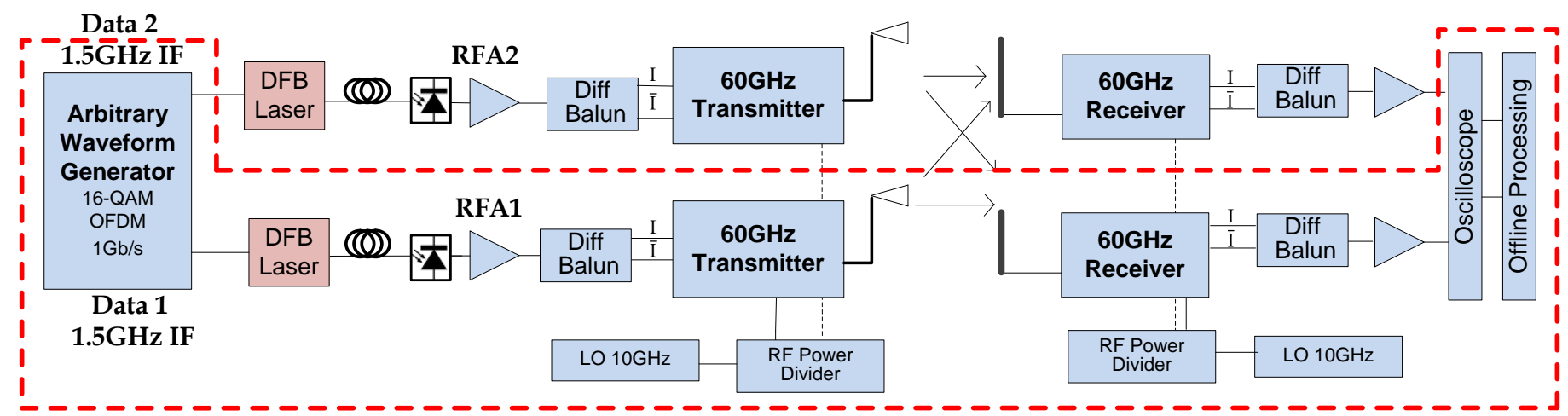

Fig.1 Experimental Setup of RoF Transport and 60GHz transmission for 2x2 MIMO (overall), for individual antenna-pair channel measurement (dashed)

signal is upconverted to $61.5 \mathrm{GHz}$, using an integrated transmitter, and wireless transmission is performed using a $20 \mathrm{dBi}$ horn antenna. At the user end, the signal is received by a $16.8 \mathrm{dBi}$ slot array antenna before being down-converted by an integrated receiver. The IF signal is amplified by an $18 \mathrm{~dB}$ gain wideband amplifier and is captured by a Digital Oscilloscope (Tektronix DPO72304DX) at the sampling rate of $12.5 \mathrm{~Gb} / \mathrm{s}$ for further processing.

The indoor measurement setup is shown in Fig. 2 where five user locations A to E were considered over an overall span of $0.8 \mathrm{~m}$. The baseline for comparison was the SISO system performance when the transmitting antenna was at a fixed location in line with the position $\mathrm{C}$. For other receiver positions, while not making any change to the tilt of the receiver antenna, the transmit antenna was tilted to ensure LOS transmission is available at each user location. For the measurements, the main limitation was angular coverage to multiple user locations and the achievable transmission distance was restricted to $1 \mathrm{~m}$ and $1.5 \mathrm{~m}$ without a V-band amplifier at the receiver side. (Larger distances can be achieved by amplifying the received signal before downconversion). In order to perform measurements for MIMO processing, the transmit power was reduced by $3 \mathrm{~dB}$. The measurements for $\mathrm{H}_{11}$ and $\mathrm{H}_{12}$ were taken by first placing the transmit antenna at locations Tx1 (transmitting Data1) and capturing the data from the receiving antenna at locations $\mathrm{Rx} 1$ and $\mathrm{Rx} 2$, respectively as shown in Fig. 2. The separation between the Rx1 and $\mathrm{R} \times 2$ locations was kept at $2.5 \mathrm{~cm}$ during the whole experiment. Then the $\mathrm{H}_{21}$ and $\mathrm{H}_{22}$ coefficients were

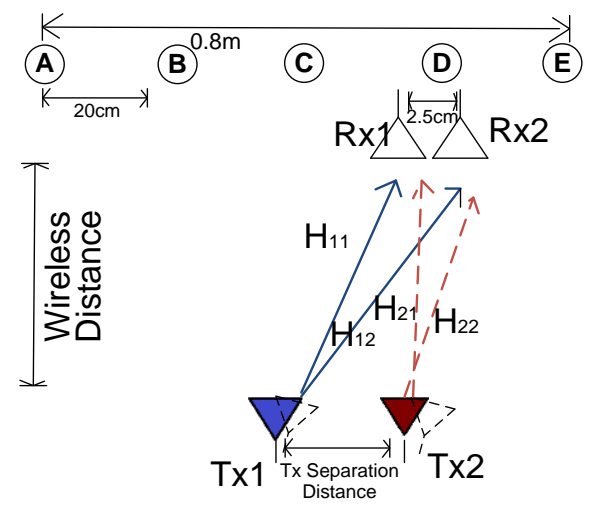

Fig. 2: Geometric orientation of experimental setup

measured by placing the transmit antenna at position $\mathrm{Tx} 2$ (transmitting Data2) and capturing the data at the two receiver locations. MIMO processing is performed offline once the channel coefficients are measured individually using this setup, which we refer to as "emulated" MIMO in this paper. To analyze the effect of spatial diversity, STBC Alamouti encoded symbols were transmitted in the form of Data1 and Data2 from the two transmit positions. In the case of spatial multiplexing, different data was sent (data rate of $0.5 \mathrm{~Gb} / \mathrm{s}$ ) from the two transmit positions and was then combined at the receive location for an aggregate data rate of $1 \mathrm{~Gb} / \mathrm{s}$.

\section{RESULTS}

The results from the SISO transmission and emulated STBC MIMO processing after wireless transmission over $1 \mathrm{~m}$ distance are shown in Fig. 3; this clearly shows that performance has been improved through spatial diversity for all user locations compared to the SISO performance, which was above the $12.5 \%$ LTE EVM limit for 16-QAM at all user locations. Fig. 4 shows similar results for $1.5 \mathrm{~m}$ transmission distance.

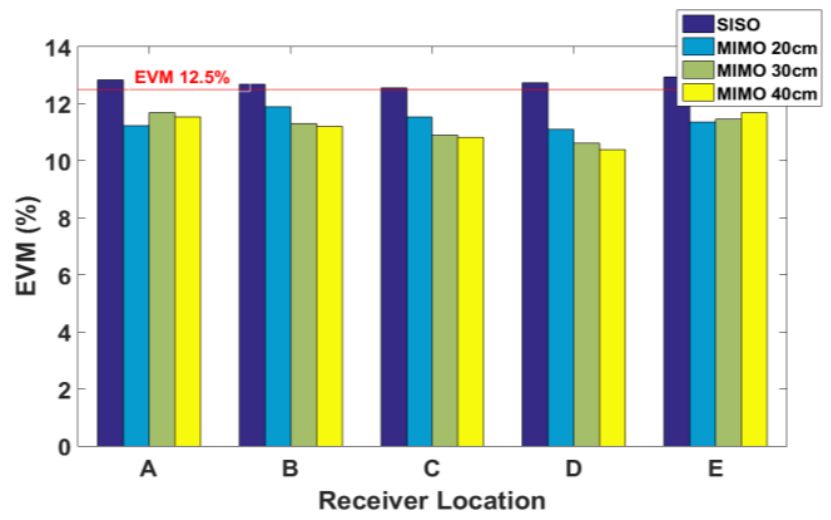

Fig. 3. Performance of STBC Alamouti emulated MIMO processing versus SISO at different user locations after $1 \mathrm{~m}$ wireless transmission 


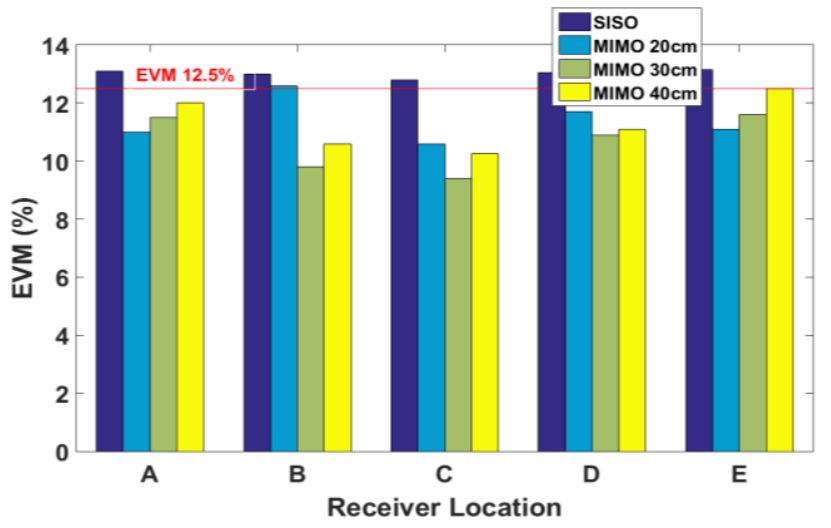

Fig. 4. Performance of STBC Alamouti emulated MIMO processing versus SISO at different User Locations after $1.5 \mathrm{~m}$ wireless transmission

The results from Zero Forcing (ZF) processing are shown in Fig. 5 and Fig. 6 for $1 \mathrm{~m}$ and $1.5 \mathrm{~m}$ wireless transmission distance, respectively. The comparison is between $0.5 \mathrm{~Gb} / \mathrm{s}$ SISO and multiplexed 1Gb/s MIMO and shows that EVM below the $12.5 \%$ limit is still achieved at $1 \mathrm{~Gb} / \mathrm{s}$ (the same overall transmit power is maintained). All of the transmit antenna separations of $20 \mathrm{~cm}, 30 \mathrm{~cm}$ and $40 \mathrm{~cm}$, provide better performance than $0.5 \mathrm{~Gb} / \mathrm{s}$ SISO transmission.

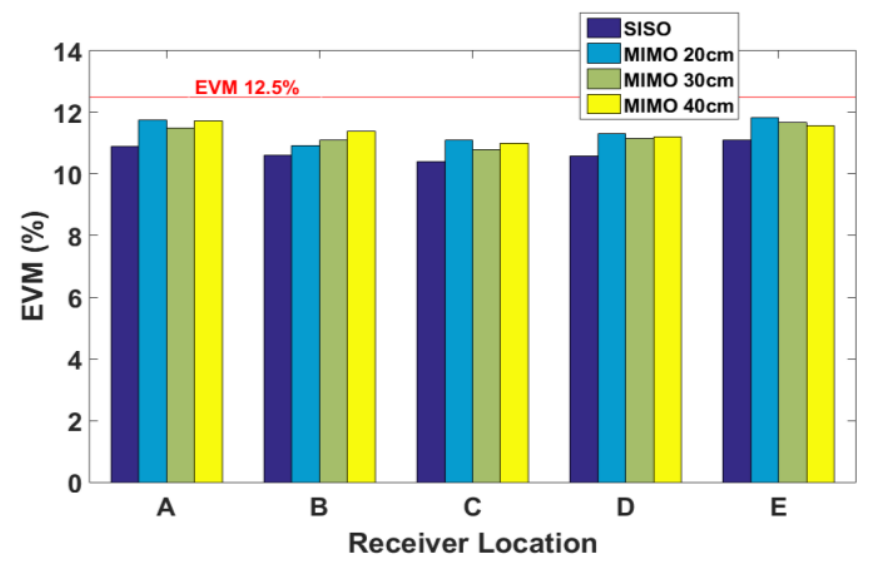

Fig. 5. EVM for SISO (0.5Gb/s) and Zero Forcing emulated MIMO reception $(1 \mathrm{~Gb} / \mathrm{s})$ after $1 \mathrm{~m}$ wireless transmission distance

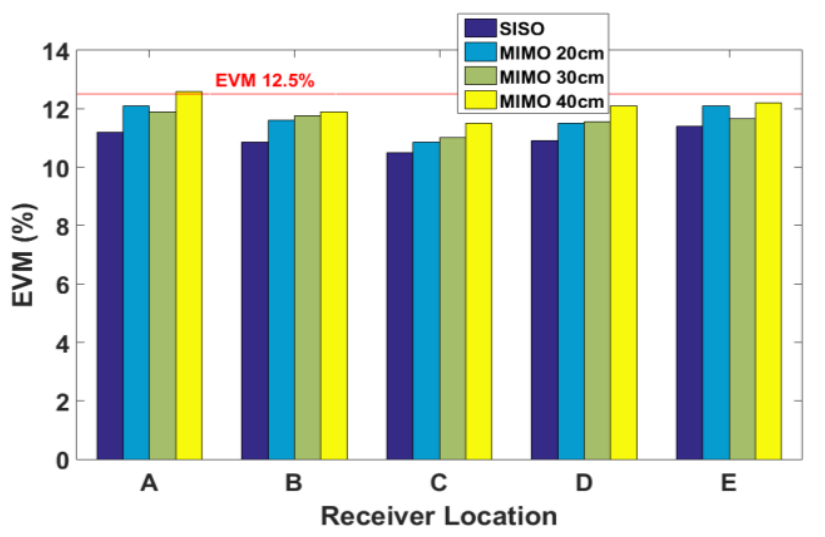

Fig. 6. EVM for SISO $(0.5 \mathrm{~Gb} / \mathrm{s})$ and Zero Forcing emulated MIMO reception $(1 \mathrm{~Gb} / \mathrm{s})$ after $1.5 \mathrm{~m}$ wireless transmission distance

\section{VERIFICATION OF RESULTS}

The results discussed in the previous section were verified by using another set of integrated $60 \mathrm{GHz}$ transmitters and receivers as shown in the complete block diagram in Fig.1. The two sets of data generated from the AWG are transported to the RAU through two RoF links. The signals at IF are upconverted to $60 \mathrm{GHz}$ by a pair of integrated transmitters and are transmitted through a pair of horn antennas. Before wireless transmission, the power was set at the same level for the two transmitting antennas by adjusting the gain of the RF amplifiers (RFA1 and RFA1 in Fig. 1) after the RoF link with a set of RF attenuators. After wireless transmission of the two signals, a pair of receiving antennas connected to the integrated receivers is used. The downconverted signals are amplified by a set of amplifiers and captured by using two channels of the oscilloscope.

The same geometrical orientation was used for the $2 \times 2$ MIMO experiments as explained in Section II and the results were compared with the emulated MIMO approach used in Section II. Fig. 7 shows the comparison of results for these real MIMO measurements and the emulated approach for STBC processing, along with the SISO performance. The wireless transmission distance was $1 \mathrm{~m}$ and antenna separation was kept at $20 \mathrm{~cm}$. It can be seen that not only do the results confirm a significant performance improvement at all user locations as compared to SISO, there is also a strong agreement between them and the emulated approach. Fig. 8 shows a similar comparison for the Zero Forcing receiver used to multiplex two $0.5 \mathrm{~Gb} / \mathrm{s}$ data signals.

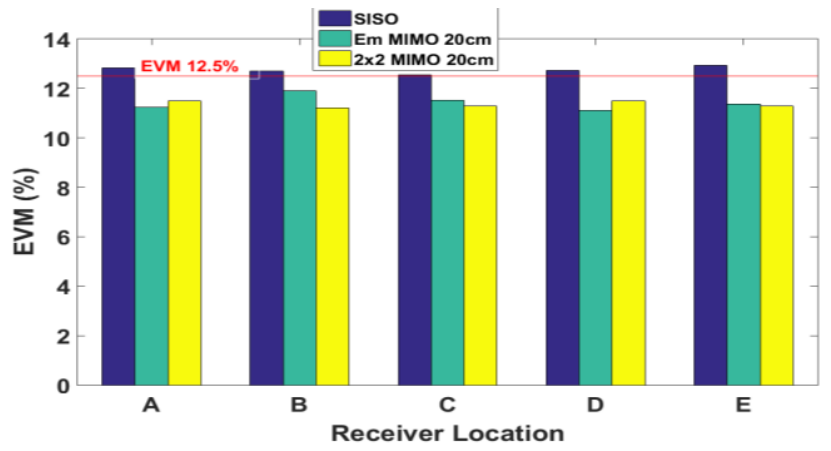

Fig. 7. 2x2 MIMO measurement results using STBC processing after $1 \mathrm{~m}$ wireless transmission distance compared to the equivalent emulated measurements and SISO performance.

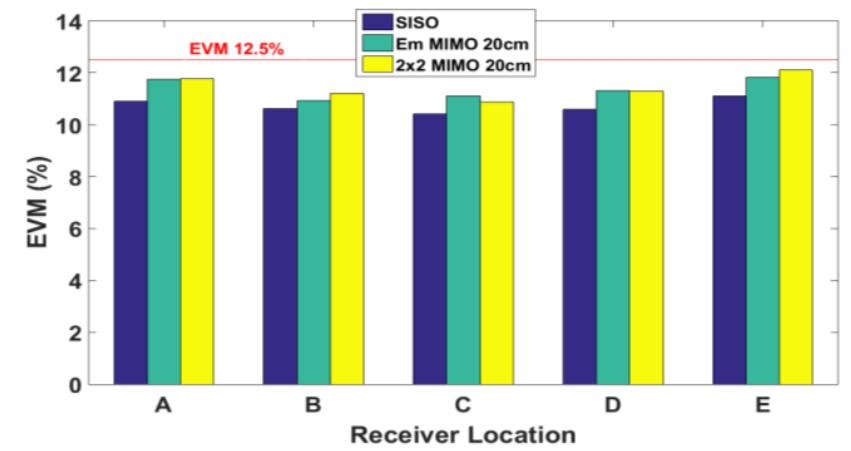

Fig. 8. 2x2 MIMO measurement results using Zero Forcing Processing after $1 \mathrm{~m}$ wireless transmission distance compared to equivalent emulated MIMO results, and SISO performance. 
Another verification has been made for the emulated MIMO measurements by observing the effect of transmit antenna spacing. An example for the transmission distance of $1.5 \mathrm{~m}$ is presented. Fig. 9 and Fig. 10 shows the effect on the EVM by increasing the antenna spacing from $20 \mathrm{~cm}$ to $30 \mathrm{~cm}$ for the emulated MIMO experiment, as well as for the real $2 \times 2$ transmission. Considering overall system performance for all user locations, performance after MIMO processing at $30 \mathrm{~cm}$ separation is better than $20 \mathrm{~cm}$ separation in both cases, and verifies the validity of the emulated MIMO measurement method. Similarly, for Zero Forcing, the best performance was observed at $30 \mathrm{~cm}$ separation distance as observed for the emulated MIMO approach.

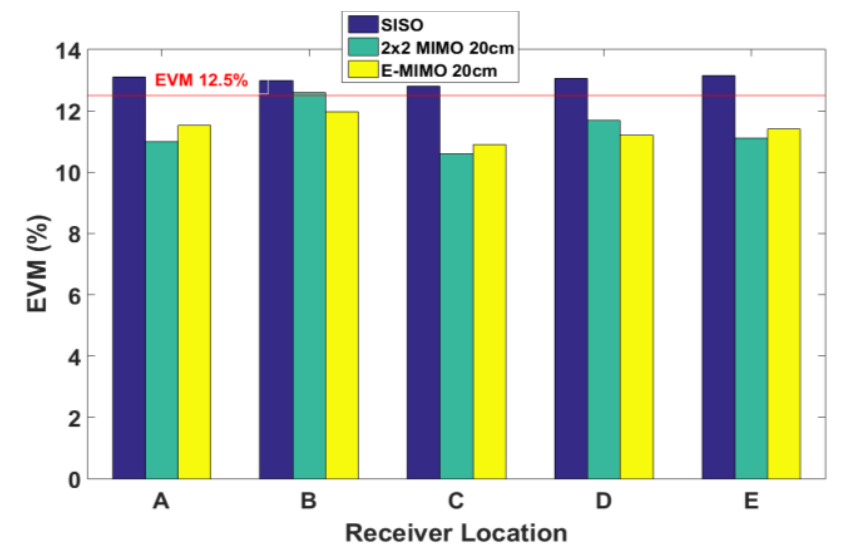

Fig. 9. Comparison of the two MIMO measurement approaches using STBC Processing with $20 \mathrm{~cm}$ Tx Antenna Separation

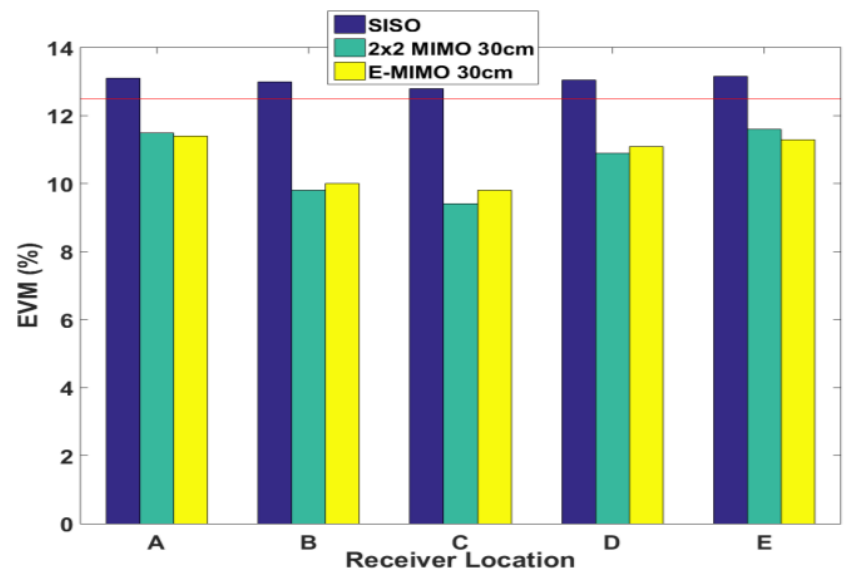

Fig. 10. Comparison of the two MIMO measurement approaches using STBC Processing with 30cm Tx Antenna Separation

A proof-of-concept for estimating mmW MIMO using analog RoF through individual channel measurements has been shown in this work and a comparison of results verifies the validity of the approach. Projections can be extended to measurements for larger system, such as $\mathrm{NxN}$, where real measurements become increasingly challenging. It could also be extended for OFDM-RoF transported mmW MIMO over longer distances and wider antennas separation, in the future work.

\section{CONCLUSIONS}

Experiments on $60 \mathrm{GHz}$ MIMO after RoF transport have been presented in this work with two measurement techniques. MIMO measurements using STBC Alamouti and Zero Forcing have been performed to achieve improvement in performance at multiple user locations. Emulation of MIMO processing through individual measurements of channel coefficients has been adopted and verified by performing MIMO with two transmitting antennas at $60 \mathrm{GHz}$ for various transmit antenna separations and hence can be projected to $\mathrm{NxN}$ MIMO measurements.

\section{ACKNOWLEDGMENT}

This work has been supported by the European Union's Horizon 2020 Research and Innovation programme under contracts 643297 (RAPID), 644526 (iCIRRUS) and the UK Engineering and Physical Sciences Research Council's NIRVANA project (Reference No. EP/L026031/1).

\section{REFERENCES}

[1] P.T. Dat, A. Kanno,T.Kawanishi, "Radio-on-radio-over-fiber: efficient fronthauling for small cells and moving cells", IEEE Wireless Communications, Vol 22(5), 2015, pp. 67-75.

[2] C.J. Hansen, "WiGiG: Multi-gigabit wireless communications in the 60 GHz band" IEEE Wireless Communications, Vol. 18(6), 2011.

[3] K. Issiali, V. Guillet, G. E. Zein, G. Zaharia, "IEEE 802.11 ac multi-user MIMO capacity and impact of antenna array geometry based on indoor measurements", IEEE 26th Annual International Symposium on In Personal, Indoor, and Mobile Radio Communications (PIMRC), 2015, pp. 726-730.

[4] L. Liu, et al, "Characterization of line-of-sight MIMO channel for fixed wireless communications", IEEE Antennas and Wireless Propagation Letters, Vol.6, 2007, pp.36-39.

[5] G. Krishnamurthy,K.G. Gard, "Time division multiplexing front-ends for multiantenna integrated wireless receivers", IEEE Transactions on Circuits and Systems I: Regular Papers. Vol. 57(6), 2010, pp.1231-1243.

[6] K. Zhu, et al, "MIMO system capacity improvements using radio-overfibre distributed antenna system technology." Optical Fiber Communication Conference and Exposition (OFC/NFOEC), 2011.

[7] A. Honda, I. Ida, Y. Oishi, Q.T. Tran, S. Hara, JI. Takada, "Experimental evaluation of MIMO antena selection system using RF-MEMS switches", IEEE 18th International Symposium on Personal, Indoor and Mobile Radio Communications (PIMRC), 2007, pp.1-5.

[8] C. Garcia-Pardo, M. Lienard, P. Degauque, J. Molina-Garcia-Pardo, L. Juan-Llácer, "Experimental investigation on channel characteristics in tunnel environment for Time Reversal Ultra Wide Band techniques", Radio Science, Vol. 47(1), 2012, pp.1-9.

[9] U. Habib, A. Aighobahi, M. Nair, H. Zhu, T. Quinlan, S. Walker, N. J. Gomes, "Performance Improvement for OFDM-RoF Transported $60 \mathrm{GHz}$ System using Spatial Diversity and Multiplexing", $10^{\text {th }}$ International Workshop on Evoloutional Technologies and Ecosystems for 5G and Beyond (WDN-5G ICC 2017), Paris, 2017. 